\title{
Characterization of JC Polyomavirus Entry by Serotonin Receptors ${ }^{+}$
}

\author{
Melissa Maginnis 1,* , Colleen L. Mayberry ${ }^{1}$, Kashif Mehmood 1 , Matthew Parent ${ }^{2}$ and \\ Samuel Hess ${ }^{2}$ \\ 1 Molecular \& Biomedical Sciences, The University of Maine, Orono, ME 04469, USA; \\ colleen.mayberry@maine.edu (C.L.M.); kashif.mehmood@maine.edu (K.M.) \\ 2 Department of Physics and Astronomy, The University of Maine, Orono, ME 04469, USA; \\ matthew.t.parent@maine.edu (M.P.); samuel.hess@maine.edu (S.H.) \\ * Correspondence: melissa.maginnis@maine.edu \\ † Presented at Viruses 2020 - Novel Concepts in Virology, Barcelona, Spain, 5-7 February 2020.
}

Published: 17 June 2020

\begin{abstract}
JC polyomavirus (JCPyV) causes a lifelong persistent infection in the kidney in the majority of the population. In severely immunocompromised individuals, JCPyV can become reactivated, spread in the central nervous system, and infect glial cells, astrocytes, and oligodendrocytes which are necessary for myelin production. The viral infection and cytolytic destruction of glial cells leads to the development of the fatal demyelinating disease progressive multifocal leukoencephalopathy (PML), for which there are currently no approved treatment options. In order to develop effective antiviral therapies, it is essential to define the virus-host cell interactions that drive infection and the virus-receptor interactions that are major regulators of tissue tropism and viral disease outcomes. Following attachment to sialic acid receptors, JCPyV requires the serotonin 5-hydroxytryptamine (5- $\left.\mathrm{HT}_{2}\right)$ receptors to mediate internalization. However, the mechanism by which JCPyV utilizes $5-\mathrm{HT}_{2}$ receptors to invade host cells is poorly understood. Using super-resolution fluorescence photoactivation localization microscopy (FPALM), we have determined that JCPyV localizes with $5-\mathrm{HT}_{2}$ receptors at timepoints consistent with viral entry. Furthermore, we have determined that the 5- $\mathrm{HT}_{2}$ receptor-associated scaffolding proteins betaarrestin, adaptor protein complex 2 (AP2) and dynamin are required for viral internalization through a clathrin-mediated endocytosis pathway. Additionally, we have identified a beta-arrestinbinding motif in the intracellular loop of the 5- $\mathrm{HT}_{2 \mathrm{~A}}$ receptor that is critical for JCPyV entry and infection. These findings highlight the importance of viral receptors in regulating viral infection and illuminate potential targets for antiviral treatment.
\end{abstract}

Keywords: JC polyomavirus; serotonin receptor; 5-HT hydroxytryptamine; FPALM; beta-arrestin; clathrin-mediated endocytosis

Funding: This research was funded by National Institute of Allergy and Infectious Diseases of the National Institutes of Health under grant number R15AI144686 and National Institute of General Medical Sciences under grant number P20GM103423. 